\title{
MORPHOMETRY OF PODOCYTES - A SINGLE CENTER STUDY OF PEDIATRIC PATIENTS: IS THERE ANY CORRELATION WITH PROTEINURIA LEVEL?
}

\section{Diana DEREWICZ ${ }^{1,2} \bowtie$, Roxana TARAS ${ }^{1,2}$, Cosmin FLORESCU ${ }^{4}$, Mihaela BALGRADEAN ${ }^{1,2}$, Maria SAJIN ${ }^{1,3}$}

${ }^{1}$ „Carol Davila“ University of Medicine and Pharmacy, Bucharest, Romania

2 „M. S. Curie“ Clinical Emergency Hospital for Children, Bucharest, Romania

${ }^{3}$ Department of Pathology, University Emergency Hospital, Bucharest, Romania

${ }^{4}$ Department of Anatomic and Molecular Pathology, National Health Laboratory, Luxembourg, Luxembourg

\section{Received 04 June 2019, Accepted 08 August 2019}

hitps://doi.org/10.31688/ABMU.2019.54.3.20

\section{Abstract}

Introduction. The most common diagnosis after a kidney biopsy in pediatric population is minimal change disease. When the prognosis is not favorable in spite of the minimal change disease diagnosis, there is a clinical suspicion of focal segmental glomerulosclerosis.

The objective of this study was to identify the ultramicroscopic changes in minimal change disease and focal segmental glomerulosclerosis focused on podocyte foot process effacement. Moreover, to find if there is a correlation between podocyte foot process effacement and proteinuria level.

Material and methods. 100 patients were included in the study: 50 in the control group, 25 in the minimal change disease group and 25 in the focal segmental glomerulosclerosis group. For each patient 5 images were taken, focused on different capillary loops to identify podocyte foot process effacement. We measured the foot process effacement related to

\section{Résumé}

Morphométrie des podocytes- étude d'un seul centre pour les enfants : y-a-t-il de corrélation avec le niveau de la protéinurie?

Introduction. Le diagnostic le plus fréquent suite à une biopsie rénale chez les enfants est la maladie à lésions minimes. Lorsque le pronostic n'est pas favorable, il y a la suspicion de glomérulosclérose segmentaire focale.

Le but de la recherche a été d'identifier les modifications ultramicroscopiques dans la maladie à lésions minimes et la glomérulosclérose segmentaire focale sur l'effacement du processus podocytaire du pied. De plus, de trouver s'il y a une corrélation entre l'effacement du proccessus podocytaire du pied et le niveau de la protéinurie.

Matériel et méthode 100 patients ont été inclus à l'étude, dont 50 dans le groupe de contrôle, 25 dans le groupe avec de la maladie à lésions minimes et 25 
glomerular basal membrane. Furthermore, we investigated the possible correlation with proteinuria using statistical analysis.

Results. The mean value of podocyte foot process effacement (\%) in the control group was $8.42 \pm 4.59 \%$, in the focal segmental glomerulosclerosis group was $55.44 \pm 16.17$, while in the minimal change disease group it was $74.35 \pm 13.01$. Compared with the proteinuria level, we established a correlation with foot process effacement ( $p$ value $<0.001)$ in the affected groups: minimal change disease and focal segmental glomerulosclerosis groups.

Conclusions. In this study, we demonstrated that there is a correlation between proteinuria level and podocyte foot process effacement in children.

Keywords: foot process effacement, proteinuria, pediatric population, minimal change disease, focal segmental glomerulosclerosis.

\author{
Abbreviations: \\ $\mathrm{FPE}=$ foot process effacement \\ GBM = glomerular basal membrane \\ $\mathrm{MCD}=$ minimal change disease \\ FSGS $=$ focal segmental glomerulosclerosis \\ $\mathrm{FPW}=$ foot process width
}

\section{INTRODUCTION}

In nowadays practice, more and more pediatric diseases are described as having a close connection with genetics or epigenetics, especially when it comes to their etiology, and in pediatric nephrology this is not an exception. Due to the major genetic discoveries in this field - genetic mutations responsible for structural and functional podocyte impairment - a new group of diseases has been described in pediatric nephrology: podocytopathies. According to Schnaper et $\mathrm{al}^{1}$, nephrotic syndrome can appear as a result of one of the following categories: diseases that are immune-mediated (through antibodies), diseases that are associated with metabolic conditions and diseases that are caused by abnormal function of glomerular cells. The last- mentioned category has its trigger in podocyte damage or dysfunction, including thus the following pathologies: minimal change disease (MCD), focal segmental glomerulosclerosis (FSGS), diffuse mesangial sclerosis, and collapsing glomerulopathy $y^{2-5}$.

One of the main features of podocytopathies is foot process effacement (FPE). However, this is not a pathognomonic characteristic of this group; it can be seen in several pathologies that are related with different levels of proteinuria. During the process of dans le groupe avec de la glomérulosclérose segmentaire focale. On a fait pour chaque patient 5 images concentrées sur différentes boucles vasculaires capillaires afin d'identifier l'effacement du processus podocytaire du pied. On a mesuré l'effacement du processus podocytaire du pied par rapport à la membrane basale glomérulaire. En outre, nous avons étudié s'il y a une éventuelle corrélation avec la protéinurie en utilisant l'analyse statistique.

Résultats. La valeur moyenne de l'effacement du processus podocytaire du pied (\%) dans le groupe de contrôle a été de $8,42 \pm 4,59 \%, 55,44 \pm 16,17 \%$ dans le groupe à lésions glomérulaires segmentaires et focales, et de 74,35 $\pm 13,01$ dans le groupe à lésions minimes. Par rapport au niveau de la protéinurie nous avons établi une corrélation avec l'effacement du processus podocytaire du pied ( $\mathrm{p}$ valeur $=<0,001$ ) dans les groupes affectés: le groupe avec la maladie à lésions minimes et le groupe avec glomérulosclérose segmentaire et focale. Conclusions. Nous avons démontré dans cette étude l'existence d'une corrélation entre le niveau de la protéinurie et l'effacement du processus podocytaire du pied chez les enfants.

Mots-clefs: effacement podocytaire, protéinurie, enfants, maladie à lésions minimes, glomérulosclérose segmentaire focale.

effacement, the 3D architecture of podocyte is massively impaired. The actin-based cytoskeleton changes its shape and it appears in electron microscopy as a thin band surrounded by nearby cytoplasm, facing the glomerular basal membrane (GBM). Usually, this phenomenon is followed by redistribution of slit diaphragm to the urinary space. Nevertheless, FPE can be a reversible or irreversible process; it depends if it's just a redistribution of components due to cytokine stimulation, such as VEGF (in this case scenario being a reversible process), or the FPE is a consequence of permanently destruction/absence of an important structural element of the cytoskeleton (therefore, being an irreversible process) ${ }^{6}$.

In pediatric population, the most common diagnosis after a kidney biopsy (usually for steroid resistant nephrotic syndrome) is minimal change disease (MCD). In Romania, $62.1 \%$ from the overall pediatric renal biopsies are diagnosed with $\mathrm{MCD}^{7}$. In this condition, the diagnosis is linked with FPE, while the number of podocytes is the same as in a healthy glomerulus. However, in FSGS, from the pathophysiological point of view, a more severe aggression is described at the glomerulus level - therefore, the involved podocytes might present FPE in an incipient phase or they can detach themselves from the GBM and undergo apoptosis. 
One of the most interesting theories is about the connection between MCD and FSGS. Both of these diseases are part of the same pathophysiological group and the differential diagnosis can be extremely challenging for a pathologist who cannot identify segmental sclerosis on a slide in order to have a $100 \%$ certain diagnosis of FSGS. This is why a lot of FSGS cases remain undiagnosed, being misinterpreted as $\mathrm{MCD}$, but from the clinical point of view the prognosis is not favorable for the patient. In these cases, usually FSGS is confirmed only if the patient has a second renal biopsy. A very interesting hypothesis states that MCD can evolve into FSGS ${ }^{8-11}$.

\section{THE OBJective OF THE STUDY}

Keeping in mind the aforementioned theory, we noticed that in „Marie Curie" Hospital, Bucharest, Romania, most of the children that underwent renal biopsy and were diagnosed with MCD, didn't have the good prognosis that was stated in the medical literature (usually MCD is in $90-95 \%$ cases steroid sensitive, some authors even consider that MCD is synonym with steroid-sensitive nephrotic syndrome ${ }^{12}$ ). Moreover, there is a lot of controversy in the medical field if whether there is or not a correlation between proteinuria level and foot process effacement, but most of these studies are based on adult popula$\operatorname{tion}^{13-15}$.

The aim of this study is to find a possible correlation between FPE and proteinuria level in two of the most common pathologies in pediatric population after a kidney biopsy: MCD vs FSGS. FPE was chosen as a variable because is one of the most intriguing features that can be seen as well as in MCD, but also in early stages of FSGS.

\section{Material AND Methods}

We designed a retrospective study (2010-2017) in which we selected pediatric patients (age $<18$ years) admitted to the Nephrology Department of „Marie Sklodowska Curie“ Hospital for Children, Bucharest, Romania, who moreover underwent renal biopsy, most of them for steroid resistant nephrotic syndrome. The renal biopsy was performed at „Carol Davila“ Nephrology Hospital, Bucharest, Romania, using the standardized biopsy gun technique with ultrasound guidance. From this group we selected those who received the following diagnoses: $\mathrm{MCD}$ and FSGS.

The control group design was based on patients who require kidney biopsy for different reasons rather than nephrotic range proteinuria (usually these patients were admitted with asymptomatic microscopic hematuria with or without low levels of protein in urine). Furthermore, to be included in the control group, one patient must not have clinical signs or symptoms such as edema, arterial hypertension or a low glomerular filtration rate based on Schwartz formula. For a more accurate statistical evidence, we assigned in the control group a patient with the same age and gender for each patient diagnosed with MCD or FSGS. Because of this, we needed to extend our database with children admitted to different nephrology hospitals but who underwent the renal biopsy at „Carol Davila“ Nephrology Hospital.

For every child involved in this study, a written consent was signed by both parents in which is stated that they are fully aware and they agree with this existing research. All the methods we used in this study are according to the national law and the ethical standards in the Helsinki Declaration of 1975, as revised in 2000. All the procedures performed in the current study have been approved by the hospital's committee for ethics based on human research.

After the biopsy was taken, the tissue was processed according to electron microscopy technique; however, it was used not only for electron microscopy study, but also for light microscopy and immunofluorescence. All the samples were fixed in $2 \%$ glutaraldehyde solution, $5 \%$ sucrose and $0.1 \%$ sodium Cacodylate buffer at a $\mathrm{pH}$ level of 7.2. For post-fixation we used a solution of $1 \%$ osmium tetroxide. After dehydration, we embedded the samples in Epoxy resins. Ultrathin sections were obtained by cutting at $0.1 \mu \mathrm{m}$; each section was stained with uranyl acetate and lead citrate.

All these sections were examined at electron microscope and for each case, 5 images were obtained according to 5 different capillary loops from a glomerulus, with a magnification power ranging from $5000-20000 x$. All the images were stored with .tiff file extension and the image analysis software that we used was ImageJ 1.52a. The length of the glomerular basal membrane in the capillary loop was measured for each image, not including the GBM facing the mesangium (just the outer part from the capillary loop facing the urinary space) and by using the same method we measured the length of the corresponding area with FPE (Fig. 1).

In this study, for better measurement accuracy, we defined FPE as being the complete effacement of all foot processes in a specific area; basically, the area in which slit diaphragm is no longer visible and the GBM is covered by a thin layer/band of completely effaced foot processes. However, from the theoretical point of view, the foot processes are not fused, they are actually retracted while the cell body of the podocyte is elongated over the $\mathrm{GBM}^{16}$. 


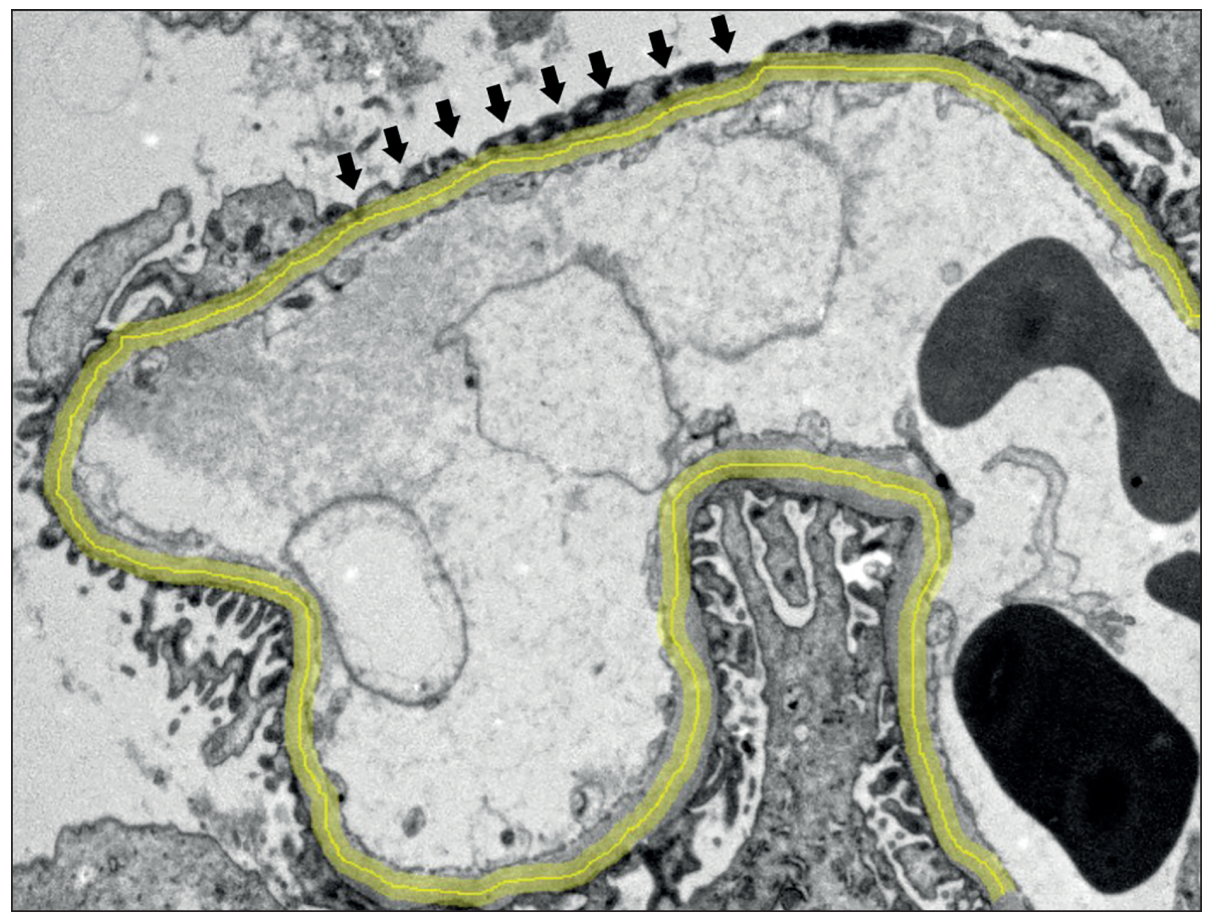

Fig. 1. The measurement of GBM (yellow line) and the area where foot processes are effaced (black arrows).

For each patient we measured FPE and GBM in 5 capillary loops and the results were expressed as percentage (\%) of FPE from GBM (FPE/GBM x 100). After that, we used the mean value of the 5 capillary loops as the final result for measurements corresponding to a single patient.

Data analysis was performed using SPSS software and Excel 2013 (especially for creating the database); the results were presented as mean value \pm standard deviation. The following statistical tests were used in this study: Student $t$ test, ANOVA / linear regression and Pearson's correlation coefficient. For a test to be statistically significant, $\mathrm{p}$ value was considered $<0.05$.

\section{Results}

In the control group we assigned 50 pediatric patients who needed renal biopsy for persistent microscopic hematuria, while in the affected group we assigned pediatric patients who underwent this procedure for steroid resistant nephrotic syndrome: 25 patients with MCD and 25 patients with FSGS. After all the measurements were done with the aforementioned method, the following results were obtained: the mean value of FPE (\%) in the control group was $8.42 \% \pm 4.59$, in the FSGS group it was $55.44 \pm 16.17$, while in the MCD group it was $74.35 \pm 13.01$ (Fig. 2).

In all of these groups, the measurements revealed different values of FPE expressed as percentage.
Therefore, Student $t$ test was run two times for better comparison between the control group vs the affected group. In each situation (comparison between MCD group and control group or between FSGS group and control group) we obtained statistically significant $\mathrm{p}$ value $(<0.001)$. Thus, we can conclude that foot process effacement doesn't occupy the same amount of space reported to GBM in the two studied groups: control group and the affected group (MCD and FSGS patients). Also, the same test was run to compare MCD group with FSGS group and we obtained a $\mathrm{p}$ value $=0.006(<0.05)$; as a consequence, the two affected groups in our study have different results of FPE length in relationship with the corresponding GBM length.

Our goal was to evaluate if there is a correlation between the level of proteinuria and the percentage of FPE in MCD group and in FSGS group. We used ANOVA test / linear regression and statistically significant results were obtained ( $\mathrm{p}$ value $<0.01$ ) in both groups (Fig. 3;4). This is related with the idea that the more protein loss in urine a patient has, the more FPE can be seen on a slide.

\section{Discussion}

After running Student $t$ test, the comparison between the control group and the affected group revealed statistically significant $\mathrm{p}$ value $(<0.001)$. This outcome was somehow expected because it 


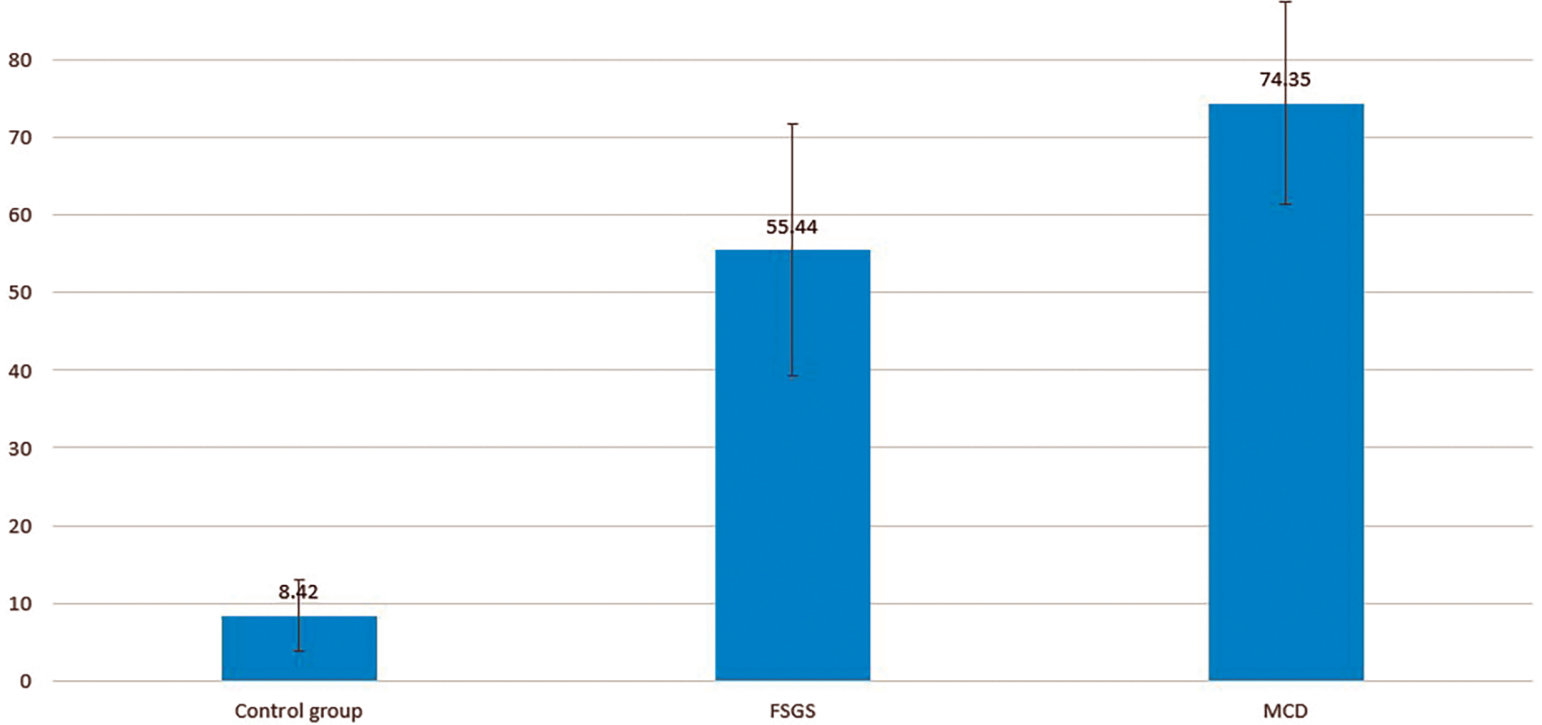

Fig. 2. Mean value of FPE (\%) in the analyzed groups

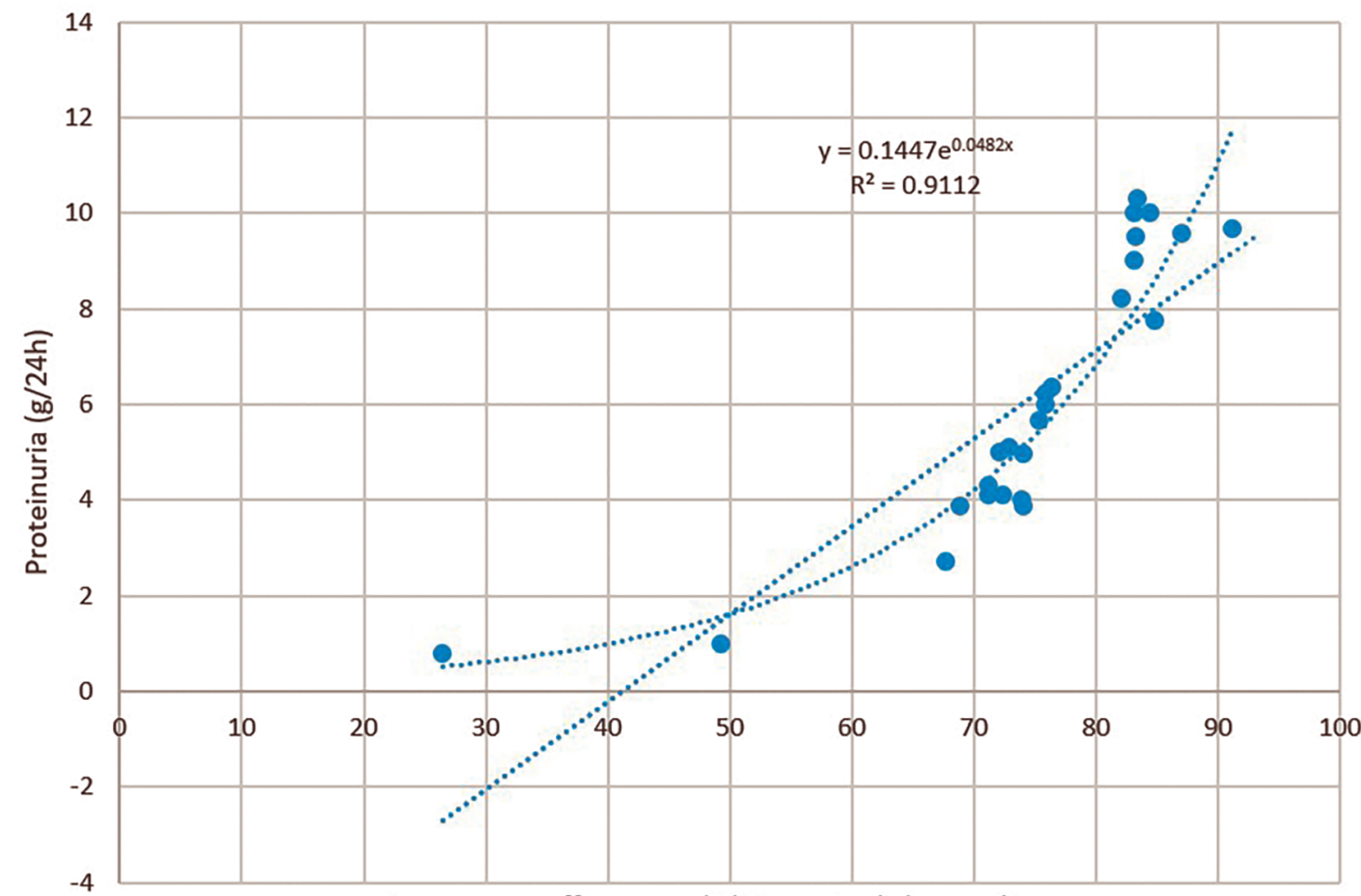

Foot process effacement (\%) in minimal change disease

Fig. 3. Linear regression (correlation between FPE in MCD and proteinuria) 


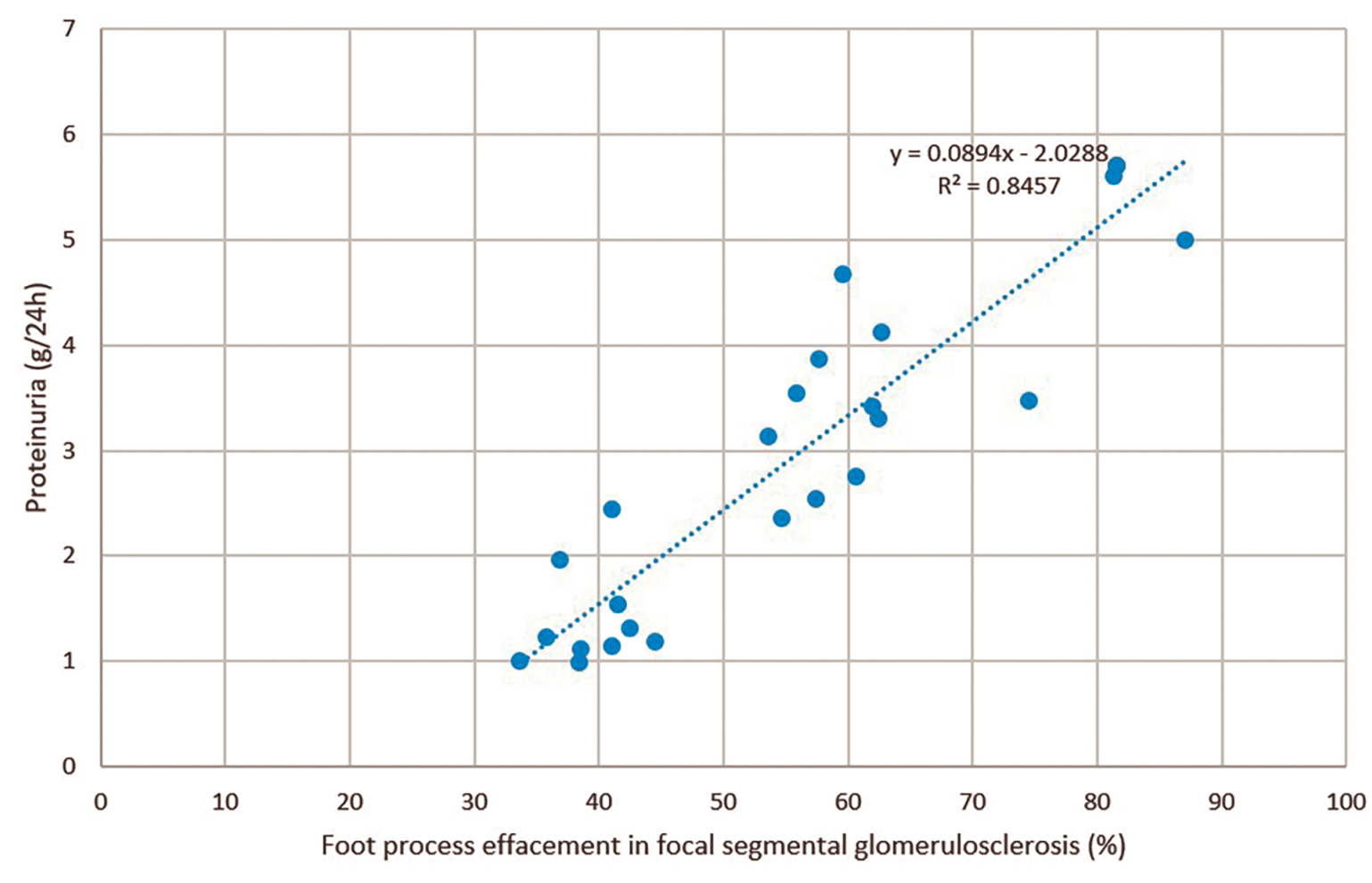

Fig. 4. Linear regression (correlation between FPE in FSGS and proteinuria)

was closely linked with the assignment in the two groups - the control group didn't include patients with heavy proteinuria; besides, FPE is well-known associated with nephrotic range proteinuria in severe glomerulopathies ${ }^{16}$.

The mean value of FPE in MCD group was $74.35 \pm 13.01$ almost the same with the one reported in the existing medical journals (however, it is stated that usually FPE is visible in an area $>75 \%$ from the capillary loop $\left.{ }^{16}\right)$. It is also reported that there can be similar degrees of fusion of the foot processes in patients with FSGS, therefore the differential diagnosis between MCD and FSGS should be settled without the extend of the fusion ${ }^{17}$. In this study we reported the two affected subgroups with statistically significant difference between them: FPE in the FSGS group was $55.44 \pm 16.17$ while in the MCD group was $74.35 \pm 13.01$. One possible explanation for this finding could rely on the fact that most of the studies are based on a comprehensive, extensive definition of FPE that includes all the degrees of fusion: from the simple narrowing of the slit diaphragm til the foot processes are no longer individualized, completely effaced. Our findings are related only with the complete disappearance (effacement) of the foot processes to facilitate an easier technique of measurement; this is why we might have missed the cases from the FSGS group with an early onset of FPE but not fully effaced foot processes.

However, other studies support the results we obtained and confirm the following hypothesis: in MCD usually the FPE is a complete mechanism, while in FSGS it doesn't reach complete effacement, but it stops in different stages of fusion ${ }^{18}$. This could be a possible explanation for the mean value of FPE that we obtained - in MCD group higher than the one in FSGS group.

Another tool of measurement worth mentioning is the foot process width (FPW). This is the most used approach the measure FPE ${ }^{19-21}$ as an indicator of foot process impairment. Basically, in the area where the foot processes are effaced, we need to measure the length of GBM and to count how many foot processes are fused (Fig. 5).

FPW is calculated with the following formula:

$$
\mathrm{FPW}=\frac{\pi}{4} \cdot \frac{\Sigma \mathrm{GBM} \text { length }}{\sum \text { foot process }}
$$

This method takes in consideration a correction factor for the angle of section through the glomerulus $(\pi / 4)$. Even if it's the most used method to calculate FPE, we considered it not suitable for our study due to the large number of images that needed to be analyzed (100 patients and for each patient we acquired 5 images with capillary loops - so a total of 500 images 


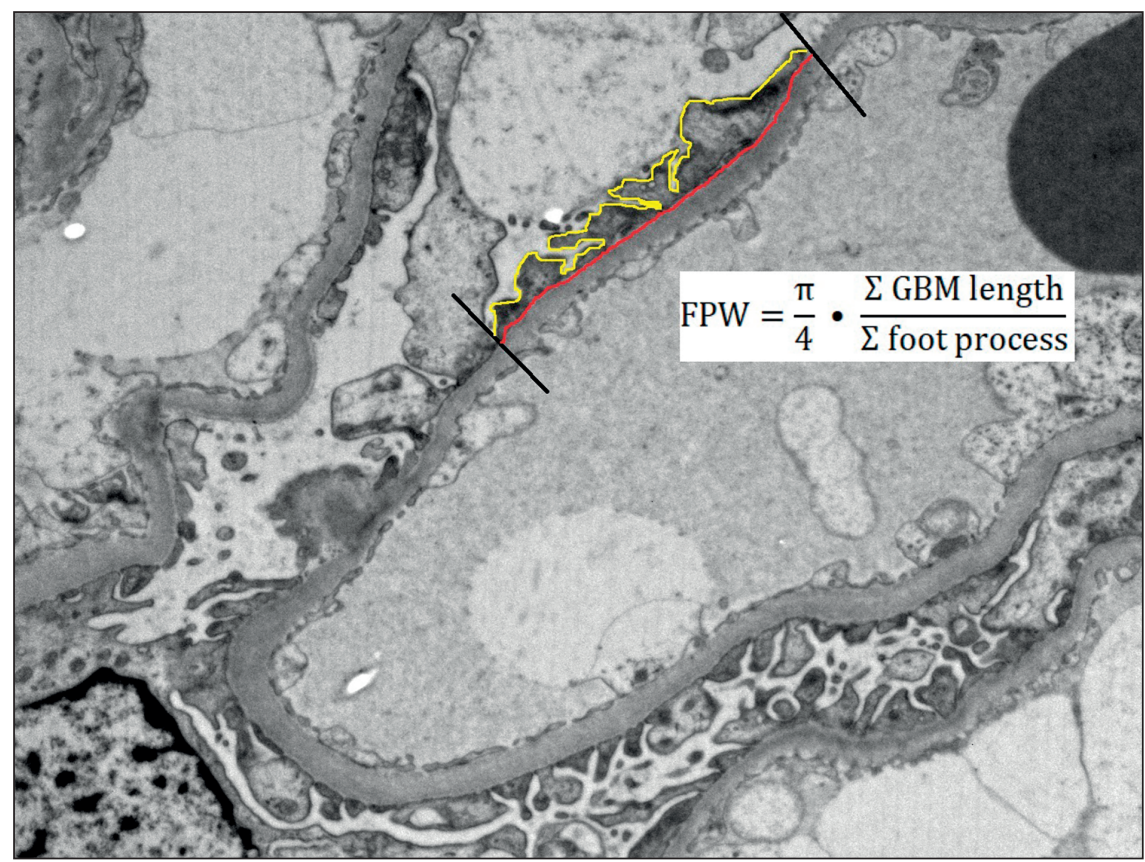

Fig. 5. The measurement of FPW in a capillary loop

to analyze). But this was not the main reason why we didn't choose this method - FPW is a perfect tool for early stages of FPE where there are still individualized foot processes but there is no longer a perfectly distinguishable slit diaphragm between them. This is required for observer to be able to count the foot processes involved in effacement. In our study we focused on complete effacement of foot processes, therefore we used the method described by Choi SY et al ${ }^{14}$ in which we measured directly the length of FPE in relationship with GBM. We consider this approach more reliable for our study and easier to use when it comes to the measurements.

The results we obtained by correlating FPE with proteinuria are interesting due to the contradiction existing in medical field about this possible association $^{13-15}$. However, the previous mentioned studies are focused on adult population or laboratory mice and our study is centered on pediatric population. This correlation is debatable because the pathophysiology of proteinuria is still under research and no relationship could be established so far between these two variables: proteinuria can be a possible cause for FPE, or on the contrary, FPE can be a possible cause for protein loss in urine ${ }^{22}$. Proteinuria can lead to podocyte impairment and thereupon to FPE, but also the process of podocyte effacement leads to architectural damage in the cell that furthermore will be responsible for protein loss in urine.

Related to studies on children, we were aware that one inconvenience that we might face takes in consideration the glomerular size and all the dimensions implied from this: FPE and GBM measurements. The kidney improves its dimensions as the child grows ${ }^{23-25}$, but we realized that it is very important to describe also pediatric population by its features, not only to imply that the studies that were run on adults can be reliable also for children.

\section{Conclusions}

In this study we established that in pediatrics, MCD patients have different values of FPE than FSGS patients. Moreover, we obtained a correlation between FPE and proteinuria levels in both investigated groups. In modern medicine, everything is mostly related to adults due to evidence-based studies while in pediatrics there is a profound lack of such research. The findings in our study are important for better understanding the specific characteristics of pediatric population with podocytopathies. However, being a single-center study, our goal is to extend the database with other Nephrology Departments from Hospitals for Children in order to validate our results to a better, more reliable statistical range, aiming for a national report in describing FPE in MCD and FSGS.

\section{Compliance with Ethics Requirements:}

„The authors declare no conflict of interest regarding this article" 
"The authors declare that all the procedures and experiments of this study respect the ethical standards in the Helsinki Declaration of 1975, as revised in 2008(5), as well as the national law. Informed consent was obtained from all the patients included in the study"

"No funding for this study"

\section{References}

1. Schnaper HW, Robson AM, Kopp JB. Nephrotic syndrome: minimal change disease, focal segmental glomerulosclerosis, and collapsing glomerulopathy, in Diseases of the Kidney and Urinary Tract, 8th Ed., edited by Schrier RW, Philadelphia, Lippincott Williams \& Wilkins, 2006, p.1585-1672.

2. Barisoni L, Schnaper HW, Kopp JB. A proposed taxonomy for the podocytopathies: a reassessment of the primary nephrotic diseases. Clinical Journal of the American Society of Nephrology. 2007;2(3):529-542.

3. Singh L, Singh G, Dinda AK. Understanding podocytopathy and its relevance to clinical nephrology. Indian Journal of Nephrology. 2015;25(1):1-7.

4. Ranganathan S. Pathology of podocytopathies causing nephrotic syndrome in children. Frontiers in Pediatrics. 2016;31:4-32.

5. Refaeli I, Hughes MR, McNagny KM. The first identified heterozygous nonsense mutations in podocalyxin offer new perspectives on the biology of podocytopathies. Clinical Science. 2019;133(3):443-447.

6. Eremina V, Sood M, Haigh J, et al. Glomerular-specific alterations of VEGF-A expression lead to distinct congenital and acquired renal diseases. Journal of Clinical Investigation. 2003; 111:707-716

7. Derewicz D, Moldovan VT, Ali, L et al. The role of glomerular morphometric features in paediatric podocytopathies a single center study. Romanian Journal of Morphology and Embryology 2018; 59(4):1061-1066.

8. Feehally J. Focal segmental glomerulosclerosis: challenges in definitions, pathogenesis and management. African Journal of Nephrology. 2017;20(1):48-56.

9. Bertelli R, Bonanni A, Caridi G, Canepa A, Ghiggeri GM. Molecular and cellular mechanisms for proteinuria in minimal change disease. Frontiers in Medicine. 2018; 5:170.

10. Maas RJ, Deegens JK, Smeets B, Moeller MJ, Wetzels JF. Minimal change disease and idiopathic FSGS: manifestations of the same disease. Nature Reviews Nephrology. 2016; 12(12):768-776

11. Müller-Deile J, Schenk H, Schiffer M. Minimal change disease and focal segmental glomerulosclerosis. Internist. 2019; 60(5):450-457.
12. Vivarelli M, Massella L, Ruggiero B, Emma F. Minimal change disease. Clinical Journal of the American Society of Nephrology. 2017; 12(2):332-345.

13. van den Berg JG, van den Bergh Weerman MA, Assmann KJ, Weening JJ, Florquin S. Podocyte foot process effacement is not correlated with the level of proteinuria in human glomerulopathies. Kidney International. 2004; 66(5):1901-1906.

14. Choi SY, Suh KS, Choi DE, Lim BJ. Morphometric analysis of podocyte foot process effacement in IgA nephropathy and its association with proteinuria. Ultrastructural Pathology. 2010; 34(4):195-198.

15. Tewari R, Nada R, Rayat CS, et al. Correlation of proteinuria with podocyte foot process effacement in IgA nephropathy: an ultrastructural study. Ultrastructural Pathology. 2015; 39(2):147-151.

16. Farris AB. Minimal change disease, Glomerular diseases (Section 2), in Diagnostic pathology. Kidney diseases, 2nd ed, Colvin RB, Chang A. Philadelphia, Elsevier, 2016, p. 48-55.

17. Fogo AB. Glomerular diseases (Chapter 3), in Diagnostic atlas of renal pathology, 3rd ed, Fogo AB, Kashgarian M. Philadelphia, Elsevier, 2017, p. 20-48.

18. Farris AB. Focal segmental glomerulosclerosis, Glomerular diseases (Section 2) in Diagnostic pathology. Kidney diseases, 2nd ed, Colvin RB, Chang A. Philadelphia, Elsevier, 2016, p. 58-72.

19. Gundersen HJ, Seefeldt T, Osterby R. Glomerular epithelial foot processes in normal man and rats. Distribution of true width and its intra- and inter-individual variation. Cell and Tissue Research. 1980; 205(1):147-155.

20. Fries JW, Rumpelt HJ, Thoenes W. Alterations of glomerular podocytic processes in immunologically mediated glomerular disorders. Kidney International. 1987;32(5):742-748.

21. Ichinose K, Kitamura M, Sato S, et al. Podocyte foot process width is a prediction marker for complete renal response at 6 and 12 months after induction therapy in lupus nephritis. Clinical Immunology. 2018; 197:161-168.

22. Kalluri R. Proteinuria with and without renal glomerular podocyte effacement. Journal of the American Society of Nephrology. 2006;17(9):2383-2389.

23. Dinkel E, Ertel M, Dittrich M, et al. Kidney size in childhood. Sonographical growth charts for kidney length and volume. Pediatric Radiology. 1985;15(1):38-43.

24. Warnakulasuriya DTD, Peries PPUC, Rathnasekara YAC, et al. Ultrasonographic parameters of the liver, spleen and kidneys among a cohort of school children in Sri Lanka. BMC Pediatrics. 2017; 17(1):192.

25. Thapa NB, Shah S, Pradhan A, et al. Sonographic assessment of the normal dimensions of liver, spleen, and kidney in healthy children at tertiary care hospital. Kathmandu University Medical Journal. 2015; 13(52):286-291. 